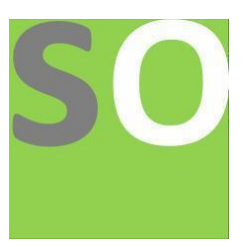

Article title: Comparative Analysis of Comic Sales Across Distinct Grades

Authors: Brian Kolo[1]

Affiliations: Magnus Partners[1]

Orcid ids: 0000-0002-3767-4995[1]

Contact e-mail: dr_loko@msn.com

License information: This work has been published open access under Creative Commons Attribution License $\mathrm{http}: / / c r e a t i v e c o m m o n s . o r g / l i c e n s e s / b y / 4.0 /$, which permits unrestricted use, distribution, and reproduction in any medium, provided the original work is properly cited. Conditions, terms of use and publishing policy can be found at https://www.scienceopen.com/.

Preprint statement: This article is a preprint and has not been peer-reviewed, under consideration and submitted to ScienceOpen Preprints for open peer review.

DOI: 10.14293/S2199-1006.1.SOR-.PPIZWRJ.v1

Preprint first posted online: 05 January 2022

Keywords: Finance, Trading, Comic 


\title{
Comparative Analysis of Comic Sales Across Distinct Grades
}

\author{
Brian Kolo
}

Dec 21,2021

\begin{abstract}
Models are created to predict the current sale price of a comic across various grades. Inputs to the models are one or more historical sale prices and their corresponding grades, and models produce a multiplier for a historical sale as an estimate of the current fair-market value. Models are realized as a table of price predictions for every possible grade. Predictions from these models average within $1 \%$ of relative value with variance of 0.07 .
\end{abstract}

\section{Introduction}

We analyze the relationship between prices of comic books at different grades. Specifically, we are interested in predicting the current value of a book in a particular grade given a recent sale of the same book in another grade.

This is a routine issue encountered with comic book collecting. Many books record only a few sales every month across different grades. This makes it difficult to accurately estimate the fair-market value for a book when there is no recent sale in the same grade.

Both buyers and sellers are interested in determining a fair price for book in a given grade and often look to historical sales to estimate the current value.

For a concrete example, suppose there were three sales of a particular comic book in the past few days: Grade 9.6 at $\$ 120$, Grade 9.4 at $\$ 100$, and Grade 9.6 at $\$ 135$. A seller has the same book graded at 9.8. Both the buyer and seller have an interest in estimating the fair-market value of the 9.8 , but there are no recent sales of the book at that grade. These models provide estimates for the current fair-market value based on these recent historical sales, reducing the uncertainties in these transactions.

Models are comprised of a set of statistical distributions for every grade pair. For ease of use, models are reduced to a simple table relating the relative price of a comic in one grade given the price in another grade. The model value for a specific grade pair is a multiplier on the price of the historical sale to obtain an estimate for the fair-market value in the desired grade.

Examining the models shows that over $23 \%$ of actual prices are within $5 \%$ of the model prediction, half fall within $12 \%$, and $70 \%$ of actual prices are within $20 \%$ of model prediction.

\section{Background}

Comic books and magazines are a form of artistic expression presented as a series of images in panels typically accompanied by a text narrative. The resulting combination is a visually inspired story often printed on a light paper containing 30-50 pages (paper quality and number of pages has varied over the past 80 years of publication).

Comic books have a long history but became increasingly popular after the publication of Action Comics 1 featuring the first appearance of Superman. This started the Golden Age of comic book in the United States (1938-1956). The Silver Age followed (1956-1970), followed by the Bronze Age (1970-1985) and the Modern Age (1985-present).

Global market size for comic books is estimated at $\$ 3.9 \mathrm{~B}$ with a CAGR of 3.3\%[3]. Individual comic books have sold at auction for over $\$ 1 \mathrm{M}$, most graded 
and authenticated by third-party independent grading companies[1].

The increase in comic popularity gave rise to comic book collecting, leading to a substantial collector market where books are sold and traded as collectables. Comic sales are often conducted via auctions on platforms such as eBay. These sales are publicly available with historical sales dating over twenty years.

Condition is a primary driver of value with comics. However, determining the condition of any collectable requires substantial expertise, and experts often disagree.

Tracking the price for collectable comic books originated with the 1965 Argosy Price Guide[2][4]. In 1970, Robert M. Overstreet started publishing the Overstreet Comic Book Price Guide on an annual basis. These were followed by additional comic price guides such as Comics Buyer's Guide (1971)[5], Wizard: The Guide to Comics (1991)[7], and Standard Catalog of Comic Books (2002) [6].

These guides estimated prices for raw books (books not graded by an independent third-party grader). Estimates were provides for various grades such as Near Mint, Very Fine, Fine, etc.

Independent comic grading companies started in the early 2000s. These companies charge a fee to review a comic book, determine its authenticity and state, and assign a numerical grade to the book. Books are then encapsulated in a clear protective case and returned.

Grading companies categorized books by label and grade. Label are Universal (most books get this label), Signature (books that are signed), Restored (repaired books), and Qualified (books with a significant defect). There are 25 grades: 10 (Gem Mint), 9.9, $9.8,9.6,9.4,9.2,9.0,8.5,8.0,7.5,7.0,6.5,6.0,5.5$, $5.0,4.5,4.0,3.5,3.0,2.5,2.0,1.8,1.5,1.0$, and 0.5 (Poor).

Various grading companies provide additional labels or combinations of labels. Some provide additional grades for single pages $(\mathrm{PG})$, no grade $(\mathrm{NG})$, or covers alone (COV).

Comic books that have not been graded by an independent grading company are termed 'raw' books. Sellers often state a nonnumerical grade that corre- sponds to (but pre-dates) the numerical grading system used by independent graders. The standard nonnumerical grades are: Mint (M), Near Mint (NM), Very Fine (VF), Fine (FN), Very Good (VG), Good (GD), Fair (FR), and Poor (PR).

\section{Sale Data}

Critical to constructing a comic price model is obtaining a set of historical sales where each book has a known grade. Comic book grades are subjective and sellers are incentivized to report grades higher than actual to increase the sale price. Buyers are incentivized to underestimate the grade to lower the price point, and the ensuing negotiation arrives at a compromised sale.

These competing incentives make it difficult to rely on grades as reported by a seller. Uncertainty in the grade of a book sold leads to uncertainty in the model for the value.

This uncertainty is reduced by examining sales of books graded by independent third-party graders. This reduces the uncertainty first by clearly stating a grade for each book, and second by utilizing grading companies and their processes to accurately state the grade.

The collector community generally debates the accuracy of third-party grading, and there are many examples where the grade on a specific book does not appear justified. However, over the several million books graded by third-party graders, these instances are relatively rare.

Transaction fees may potentially bias the sale price. Auction houses typically charge fees as a percent of the sale price, potentially with a minimum fee. Fees may be assessed to the buyer, seller, or both. This structure varies over different auction houses and this may bias the final sale price as buyers may incorporate these fees into their maximum bids.

Collecting and curating sales data is error-prone for several reasons. Transcription errors may result in incorrect prices, grades, dates, and titles. Additionally, unscrupulous sellers and/or buyers may introduce fraudulent transactions into the data stream in an attempt to influence market prices. 
Incorrect transactions records may affect the quality of the models produced. Some records may be traced back to primary sources whereby the transaction record can be compared to the primary source and corrected.

This section reviews the process used to select sales records used for modeling. Historical sales data for graded comic book contain sales from multiple auction platforms with books graded by different thirdparty graders. Potential variance between platforms and graders is reduced by limiting the data analyzed to a single auction platform and books graded by a single third-party grader.

\subsection{Single Third-Party Grader}

We only consider sales of books graded by Certified Gratuity Company (CGC). Using a third-party grade reduces the uncertainty for the grade of the underlying book. This also assigns numerical value to the grade which has higher fidelity then the nonnumerical grades typically used for raw books (25 numerical grades vs. 8 nonnumerical grades).

Certified Gratuity Company (CGC) is the single third-party grader considered. First, choosing a single third-party grader eliminates variance in grade assessment between different grading companies. Grading companies employ different processes and may have different grading standards. This reflects the subjective nature of grade determination in the collectables market.

Second, CGC is chosen because the majority of graded books sold were graded by CGC. Choosing CGC provides the largest possible set of data with a single grader.

Third, CGC is one of the oldest grading companies providing potentially twenty years of historical sales. Analyzing a long history provides the opportunity to minimize effects of recent trends.

\section{$3.2 \quad$ Universal Label}

Only books graded as 'Universal' are considered. Universal labels comprise approximately $90 \%$ of sales records. Limiting analysis to these records does not substantially reduce the number of records under consideration. Other labels are distinguished from Universal and likely have a different value from their Universal counterparts at the same grade.

Signature Signature labels typically have increased value over Universal labels because having books signed is considered an enhancement. This creates an upward bias on the price of the book relative to a Universal book at the same grade.

Qualified Qualified books have a significant defect which requires description. These defects may materially impact the value of the book.

Restored Restored books are generally considered less desirable than unrestored books. The effect on value likely varies by the individual book under consideration.

\subsubsection{Single Platform}

Only sales from the eBay platform are considered. Different auction houses have different costs to buyers and sellers. Some auction houses charge fees to both buyers and sellers, while others only assess fees to sellers. Some auction houses charge buyers a minimum fee meaning and a percentage above the minimum while others do not charge buyers fees for any transactions.

These differences have the potential to skew the sale price from the fair-market value. Choosing a single platform reduces this effect, and at the very least produces a consistent bias to all transactions.

eBay is chosen because most of the data under analysis originated from this platform. Over $90 \%$ of graded comic sales arise from the eBay platform.

\subsection{Initial Selection}

The initial selection of comic sales data contains approximately 650,000 sales records, with over 500 different comic books, and sales over a period of 20 years. Limiting to CGC Universal books sold on eBay results in over 500,000 sales records.

The books selected are among the most often transacted comic books. These books are regularly sold 
across various grades and are highly desired by collectors.

The books selected sample from the Golden Age through the Modern Age, between several publishers, grades, topics, and series. This provides a broad base for a general analysis of sale prices against grades.

\subsection{Cleaning}

Entries in the time-series data sequence may individually display anomalous sales values. These may arise from negligent handling of the data, or from intentional bad actors.

For example, the sales data is transcribed multiple times prior to arriving at its final destination. Transcription errors may alter values, and automated ETL techniques may incorrectly identify the grade or title of a book. These negligent acts may corrupt a substantial number of records.

Alternatively, bad buyers and sellers may conspire to create specious transactions attempting to raise or lower the expected sale price. These intentional acts also corrupt the time-series and lead to long-tailed distributions.

The following reviews several means whereby the time-series data may become corrupted.

Incorrect Grade Assigned The grade assigned in the time-series is incorrect. The dataset may include images of the actual item sold and checking this image reveals that the CGC grade on the book in the image does not match the text value for the grade in the dataset.

Incorrect Title The title of the book is incorrect. Similar to above, examining an image associated with the sale reveals that the title per the dataset does not match the actual title of the book sold.

Incorrect Price The sale price of the book is incorrect. This may be rectified if the sales record can be traced back to a primary source.

Incorrect Date The date of the sale is incorrect. Similar to Incorrect Price, this may be rectified if the sales record can be traced back to a primary source.
Multiple Items in Lot Here the dataset reports the sale of a particular book in a specific grade, but detailed examination reveals that there were in fact multiple items sold as a single lot. These records are removed as the sale price is not attributed to a single book.

False Sale This is the case of a fake sale. A prospective seller may create an auction listing at a price much higher than historical transactions, then purchase the item with another account, hoping to temporarily raise the price of the item then selling their item at the inflated price.

False Purchase Similar to the previous, a prospective buyer creates an auction listing at an artificially low price then buys the item with another account, hoping to temporarily reduce the price and take advantage of the price reduction in another purchase.

Shill Bidding This is another means of a Fake Sale. Here a seller creates an auction listing then uses two different accounts to bid against each other raising the price to artificially high levels[8].

\subsubsection{Cleaning Process}

The goal of cleaning the time-series is to separate incorrect sales records from valid sales. This is possible for some sources of error such as Incorrect Grade Assigned, Incorrect Title, and Multiple Items in Lot. When these records are associated with an image, a manual check of the data can identify incorrect sales records.

However, False Sales and Shill Bidding cannot be determined with complete confidence. Valid sales may appear fraudulent and fraudulent sales may appear valid. There is an active body of research examining online sales to identify these cases[12][10][15].

Presently, time-series is cleaned by examining the historical sale price of a given book in a specific grade. A 30-day time window is used, and an individual sales record is compared to the average price of other sales (same book, same grade) within the window. From this 30-day time series, the z-score of the sales record 
under consideration is computed. Sales outside a critical value of the z-score are rejected and removed from the time-series.

A 30-day window is a relatively short period for comic sales. Prices typically do not vary much over 30-days, and sales here are generally trading rather than trending. Sales within this window are considered to occur close enough together to be considered measurements at nearly the same moment.

The z-score is used as an indicator of unusual sale prices and outlying records are removed from the time-series. The process proceeds as a series of steps:

Group by Title-Label-Grade Time-series records are grouped by Title, Label, and Grade. This results in 23,342 separate time series.

Time-Windowed For each sales record, find all sales 15 days before and after. The result is a subset of a Label-Title-Grade series containing all sales within a 30-day window, except the sale under consideration.

Average \& Variance Compute the average and variance for the data in the windowed subset. Use a minimum variance of 0.05 when there is only one point in the subset or when the variance of the subset is below 0.05 .

Compute the Z-Score Compute the Z-score of the sale under consideration using the average and variance from the previous step.

Filter Z-Score Remove records with a z-score outside a specified range (the results of possible ranges are discussed below).

This process discards sales records that do not have at least one other sale with the same Label-TitleGrade within the 30-day window. The orphans $44.6 \%$ $(252,712)$ of the sale records for Universal Labels sold on the eBay platform $(566,185)$. These orphan sales are considered for some models as discussed below.

Using the z-score as the measurement of interest normalizes each of the time-series to a dimensionless variable. These measures are combined into a single time-series replacing their corresponding sales value from the original time-series.

Figure 1 shows the relationship between the percentage of records retained as the z-score window is increased. $90 \%$ of the records fall within a z-score of 3.5 while $2.5 \%$ of records lie outsize a z-score of 10 .

The average, variance, skewness, and kurtosis are computed from this time-series when different values of the critical z-score window are used. Figure 2 shows the relationship between the average and variance of the time-series at different values of the zscore cutoff. Similarly, Figure 3 depicts the relationship between the skewness and kurtosis.

The $\beta_{1}-\beta_{2}$ relationship shows the time-series distribution is near Normal when the z-score window is between 2.5-3.5. As the z-score cutoff is increased, the distribution moves into the Pearson IV family[13]. The heavy tail of the sales data rapidly increases the kurtosis as outliers are included.

Based on this, a z-score cutoff in the range $(2.5,3.5)$ is recommended. However, it will be shown below that the resulting models are insensitive to this selection and produce similar results even when including nearly all sales data.

Figure 2 shows a scatter plot of the average and variance of the resulting distributions with a z-score cutoff on the range $[0.25,10]$. The average of the $z^{-}$ scores is near zero for all z-score cutoffs. The variance has a larger range and is less than 2 up to a cutoff of 5 .

Figure 3 is a Pearson plot of $\beta_{1}$ (squared skewness) and $\beta_{2}$ (kurtosis). The distribution is near normal for cutoffs in the range $[2,4]$. Lower values for the cutoff indicate Pearson Type I distributions (Beta Distributions) while higher values move increasingly into the Pearson Type IV family.

Larger values of the cutoff include more extreme values and pull the distribution farther into the Pearson Type IV family. Cutoff values in the range $[2,4]$ cluster near Normal, while the corresponding average is near zero and variance is near one. Taken together it is reasonable that the clean distribution is near Normal and a cutoff of 3 has average, variance, skewness, and kurtosis all near expected values. 
Sales Retained vs. Z-Score Window

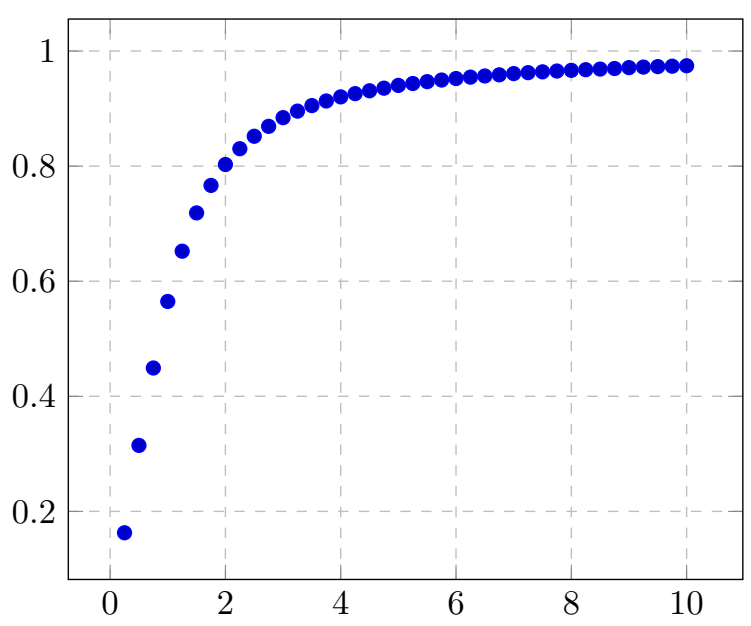

Figure 1: Percent of sales records remaining versus z-score cutoff.

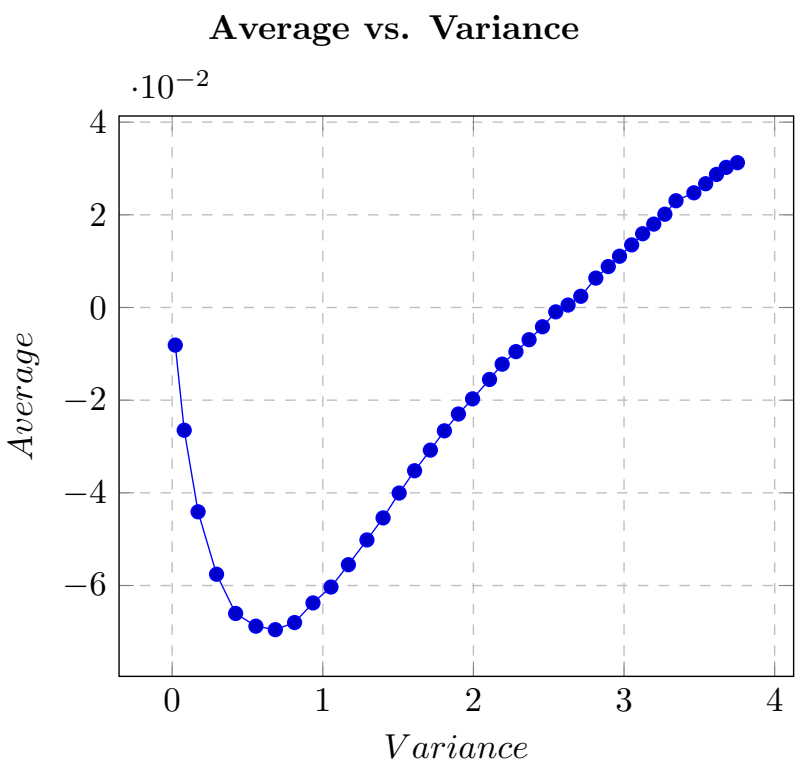

Figure 2: Scatter plot of Average and Variance for z-score windows ranging from 0.25 to 10 .

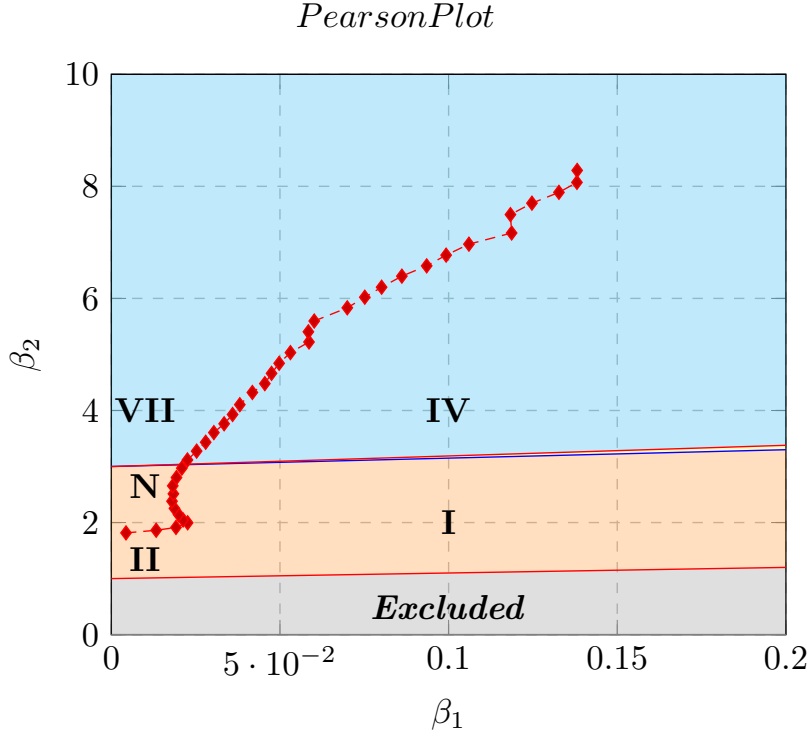

Figure 3: Pearson plot for z-score windows from 0.25 to 10 .

\subsection{Sale Pairing}

Analysis of the data compares the sale prices of two records where an earlier in time record is used to predict the value of the later record. This analysis requires records to be pairs together.

Records may only be paired if they are within the same comparison window. This is a window of time when sale prices are not expected to vary.

This window is distinct from the 30-day window used with the z-score, although the purpose is somewhat similar. The 30-day window used with the $\mathrm{z}-$ score is the time period where a sale is compared to similar sales, and statistical outliers are eliminated. An individual record is compared with sales 15-days in the future and 15-days in the past. The 30-day window is centered on the sale under consideration, and this sale is compared with sales that have the same Title-Label-Grade.

The comparison window is a look-back period from the sale under consideration. We are interested in predicting the current price for a particular TitleLabel (but not Grade), and all historical sales are considered. The comparison window measures how 
far back in time past sales may be used to predict the current value.

Paired records share the same title, but need not be the same grade. This allows analysis of the grade dependence on the price which is the essential feature under analysis.

There are two approaches we take in data pairing:

Single-Pared Sales In Single-Pared Sales, each sale appears in no more than one pair. A record is paired with the nearest earlier record, if one exists, in the comparison window. Neither this record nor the paired record appear in any other pair. Model parameters computed under this pairing method are sourced from independent unrelated sales. The number of pairs is at most half the number of sales records.

Multi-Pared Sales In Multi-Pared Sales, each record is paired with all other previous records within the comparison window. These records may appear in multiple pairs and can contribute to the analysis in multiple ways. Model parameters computed under this method may be correlated with each other because the parameters are computed using the same sales records. This number of pairs here is much larger and could be as large as $N(N-1) / 2$ with $N$ sales records.

\subsection{Datasets}

We arrive at four datasets for analysis, depending on whether the sales are Single- or Multi-Paired, and whether orphaned sales are kept or discarded.

The analysis in the next section compares results for each of the four datasets. These are described here for each of later reference:

Single-Paired Exclude Orphans (SEO) Sales appear in at most one sales pair and orphan records (records without another sale in the same grade and title) are excluded.

Single-Paired Include Orphans (SIO) Sales appear in at most one sales pair and orphan records are included.

\section{Multiple-Paired Exclude Orphans (MEO)}

Sales are paired with all previous sales within the comparison window, and orphan records (records without another sale in the same grade and title) are excluded.

\section{Multiple-Paired Include Orphans (MIO)}

Sales are paired with all previous sales within the comparison window, and orphan records are included.

\section{Model}

A multiplicative model is used to predict the values of a grade based on a sale in another grade. The model is driven as the relationship

$$
\mathcal{M}\left(G_{1}, G_{2}\right)=\frac{F M\left(G_{1}\right)}{F M\left(G_{2}\right)}
$$

where $F M(G)$ represents the fair-market value for the title in grade $G$.

Given the current price of a book graded $G_{2}$, the model price in another grade $G_{1}$ is simply the product $F M\left(G_{1}\right)=F M\left(G_{2}\right) \mathcal{M}\left(G_{1}, G_{2}\right)$.

A model is a set of distributions of relative prices for a specific grade pair where the first element of the grade pair is the grade of the prediction and the second element is the grade of a historical sale. A comparison window is used to determine the range of historical sales that may be used to predict a current sale price.

For every sale in the time-series, we select a set of historical sales, compute the relative price, and add this to the distribution. The selection process may include all sales in the comparison window (MultiPaired) or it may only include the most recent sale (Single-Paired).

Applying this to the entire time-series we arrive at a set of up to 625 distributions (every grade may be paired with every other grade). Not all grade pairs are present in the time-series, so the number of distributions present for a given model is typically less than 625. Multi-paired models typically have data on 400-450 distributions while single-paired models contain 350-400 distributions. 
Distributions arising from single-paired models are based on independent data. Every sales record contributes to no more than one distribution.

Multi-paired models may have a single sales record contribution to multiple distributions. Every sales pair matches to a model distribution, so multi-paired datasets may have a singe sales record associated with many distributions.

We compute 1,220 different models by examining four different datasets (SIO, SEO, MIO, MEO) against five different $\mathrm{z}$-score windows $(2.00,2.75$, $3.00,5.00,10.00)$ over 61 comparison windows (0-60 days inclusive). Each of these models is run with individual cross-validation on the time-series resulting in $1,102,575,644$ cross-validation models, each providing a single test of the corresponding model.

\subsection{Time-Independent Model Con- straint}

If the model from 1 is considered time-independent, then a constraint arises between grades. Timeindependence requires a symmetry between the predictions as discussed below.

Specifically,

$$
\begin{aligned}
\mathcal{M}^{-1}\left(G_{1}, G_{2}\right) & =\left(\frac{F M\left(G_{1}\right)}{F M\left(G_{2}\right)}\right)^{-1} \\
& =\frac{F M\left(G_{2}\right)}{F M\left(G_{1}\right)} \\
& =\mathcal{M}\left(G_{2}, G_{1}\right)
\end{aligned}
$$

Generally, the model from 1 is constrained by intermediate grade chains:

$$
\begin{aligned}
\mathcal{M}\left(G_{1}, G_{3}\right) & =\frac{F M\left(G_{1}\right)}{F M\left(G_{3}\right)} \\
& =\frac{F M\left(G_{1}\right)}{F M\left(G_{2}\right)} \frac{F M\left(G_{2}\right)}{F M\left(G_{3}\right)} \\
& =\mathcal{M}\left(G_{1}, G_{2}\right) \mathcal{M}\left(G_{2}, G_{3}\right)
\end{aligned}
$$

This must be true for any intermediate grade $G_{2}$. The process may be continued to higher order products, however, if the model satisfies the constraint in 3 all higher order constraints are satisfied as well.
This relationship reduces the number of independent parameters in the model, and we seek the minimum number of independent parameters in the Model. The model covers all 25 distinct grades creating 625 paired entries for $\mathcal{M}(x, y)$.

Examining the constraint

$$
\mathcal{M}(x, z)=\mathcal{M}(x, y) \mathcal{M}(y, z) \forall x, y, z:
$$

Case 1: $x=y=z \mathcal{M}(x, x)-\mathcal{M}^{2}(x, x)=0 \Longrightarrow$ $\mathcal{M}(x, x)=\operatorname{0or} \mathcal{M}(x, x)=1$. Only the latter case leads to non-trivial models.

Case 2: $x=z \neq y \mathcal{M}(x, y) \mathcal{M}(y, x)=1$.

Case 3: $x \neq y \neq z \mathcal{M}(x, z)=\mathcal{M}(x, y) \mathcal{M}(y, z)$

Let $N$ represent the number of distinct grade values for the model. The model $\mathcal{M}(x, y)$ has $N^{2}$ total parameters. These parameters may be viewed as entries in a matrix, but a more convenient representation is a complete graph on $N$ vertices with loops.

Figure 4 shows a graphical representation of the model on five nodes. Vertices represent grades while directed edges represent parameters in the model. Edges are bidirectional between nodes indicating $\mathcal{M}(x, y)$ and $\mathcal{M}(y, x)$ are separate parameters.

The constraints reduce the number of independent parameters. Case 1 fixes $N$ parameters $\mathcal{M}(x, x)=1$. These are the loops in the graph. Case 2 determines $\mathcal{M}(y, x)$ in terms of $\mathcal{M}(x, y)$, rendering the arrows in the graph unnecessary. Case 3 writes one grade pair in terms of the product of related grade pairs. This is a triangle in the graph, and the constraint means there can be only one path between nodes.

Taken together the constraints reduce the number of parameters to $N-1$. Graphically, the choices of the independent parameters are the spanning trees on the complete graph.

The number of spanning trees for a complete graph with $N$ labeled nodes is given by Cayley's formula $N^{N-2}[9]$.

The choice made here is the chain of successive grades. In this case the independent parameters are the model values $\mathcal{M}(10,9.9), \mathcal{M}(9.9,9.8)$, $\mathcal{M}(9.8,9.6), \ldots \mathcal{M}(1.5,1.0), \mathcal{M}(1.0,0.5)$. 


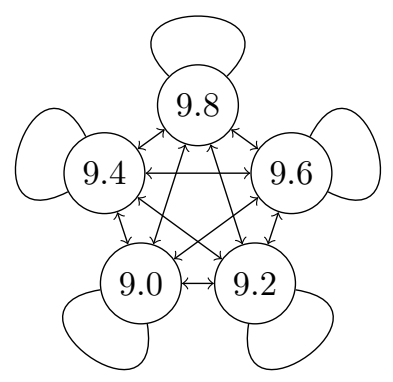

Figure 4: Complete directed graph on five nodes with loops.

Figure 5 provides an example of a spanning tree for the complete graph on five nodes. The independent parameters are the edges of the graph: $\mathcal{M}(a, b)$, $\mathcal{M}(a, c), \mathcal{M}(a, d)$, and $\mathcal{M}(a, e)$. All other model parameters may be written in terms of these (see 1 ).

For the full model we have $N=25$, and there are $1.42 \times 10^{3} 2$ different sets of independent parameters. Among these we choose the one which maximizes the number of sales records between the independent parameters. This choice maximally utilizes the sales records used to compute the independent parameters of the model.

To optimize this, assign to each edge in the complete graph the number of records associated with the corresponding parameter. Each spanning tree is assigned a value by summing the values assigned to each edge.

Spanning trees of a connected graph form a matroid[14], and Kruskal's algorithm computes the optimal edge weighted graph among the spanning trees[11]. Kruskal's algorithm employs a greedy approach one edge at a time, adding the maximally weighted unused edge if that edge does not create a cycle.

\subsection{Time-Dependent Models are Un- constrained}

The constraint detailed in the previous section assumes the model is time-independent. Time-

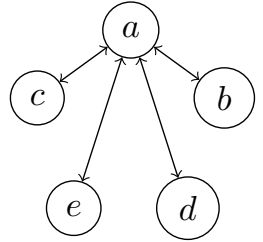

Figure 5: Example of a spanning tree on five nodes.

\begin{tabular}{|c|ccccc|}
\hline & $a$ & $b$ & $c$ & $d$ & $e$ \\
\hline \hline a & 1 & $M_{a b}$ & $M_{a c}$ & $M_{a d}$ & $M_{a e}$ \\
b & $M_{a b}^{-1}$ & 1 & $M_{a b}^{-1} M_{a c}$ & $M_{a b}^{-1} M_{a d}$ & $M_{a b}^{-1} M_{a d}$ \\
c & $M_{a c}^{-1}$ & $M_{a c}^{-1} M_{a b}$ & 1 & $M_{a c}^{-1} M_{a d}$ & $M_{a e}^{-1} M_{a e}$ \\
d & $M_{a d}^{-1}$ & $M_{a d}^{-1} M_{a b}$ & $M_{a d}^{-1} M_{a c}$ & 1 & $M_{a d}^{-1} M_{a e}$ \\
e & $M_{a e}^{-1}$ & $M_{a e}^{-1} M_{a b}$ & $M_{a e}^{-1} M_{a c}$ & $M_{a e}^{-1} M_{a d}$ & 1 \\
\hline
\end{tabular}

Table 1: Model parameters in terms of the independent parameters from Figure 5.

dependent models are not subject to this constraint.

The model is used to predict a future sale price given a previous sale. In the time-dependent model, the fair-market functions must be labeled as past or future. Rewriting 1,

$$
\mathcal{M}\left(G_{1}, G_{2}\right)=\frac{F M_{\text {past }}\left(G_{1}\right)}{F M_{\text {future }}\left(G_{2}\right)}
$$

Reexamining the constraint from 2 ,

$$
\begin{aligned}
\mathcal{M}^{-1}\left(G_{1}, G_{2}\right) & =\left(\frac{F M_{\text {past }}\left(G_{1}\right)}{F M_{\text {future }}\left(G_{2}\right)}\right)^{-1} \\
& =\frac{F M_{\text {future }}\left(G_{2}\right)}{F M_{\text {past }}\left(G_{1}\right)} \\
& \neq \mathcal{M}\left(G_{2}, G_{1}\right)
\end{aligned}
$$

The constraint does not hold, and the model parameters are independent of each other.

\subsection{Time-Dependent Models}

\subsubsection{Selection of Dataset}

The Multi-Paired Exclude Orphan (MEO) dataset is selected. Figures 4.3 .1 and 6 compare the performance of the four models across comparison windows form 0 to 60 days. 
The model variance is a useful measure of the dispersion of predictions, while the absolute average aids in understanding how far the relative predictions are from the true values. Both the variance and absolute average are measuring against the relative error of predictions against the true sales value. A (-) $5 \%$ relative error means the true value was $5 \%$ above(below) the predicted value.

Reviewing these figures we see the Multi-Paired Exclude Orphan dataset has better performance in both measures. The MEO model has consistanly lower variance and the absolute value of the relative difference is lower in all cases except the SEO model at a zero-day comparison window.

A multiobjective optimization approach is also undertaken. Figure 7 provides a multiobjective optimization plot of the four models with the Utopia point at $(0,0)$ and the Nadir point at (inf,inf).

Two incomparable models remain: Multi-Paired Exclude Orphan model on a 15-day window and a Single-Paired Exclude Orphan 0-day model. The 15day MEO model provides the opportunity for many historical prices to contribute to the model prediction. A 0-day model is impractical as it constrains the time to make the prediction and determine how to act. However, this model may be useful in automated trading environments and may be further considered in later work.

The multiobjective approach leads to two optimal datasets, but we choose a closely related dataset as the basis for modeling. The MEO 14-day dataset is very close to the MOO optimial MEO 15-day, but has the advantage of containing two complete weeks of sales. This minimizes the day-of-the-week impact from the time-series.

The graph of model variance includes an interesting feature useful in identifying incorrect sales in the time series. Sales that are far outside the typical range cause the variance to jump when the comparison window is large enough to include an extreme value. Models analyzing orphan sales are particularly sensitive as the orphan sales are not subject to the cleaning process with the rest of the time-series.

One example may be seen in Figure 4.3.1. The MIO model variance slowly changes as the comparison window approaches 20 days, then jumps quickly.

\section{Model Variance of the four models as a function of comparison window.}

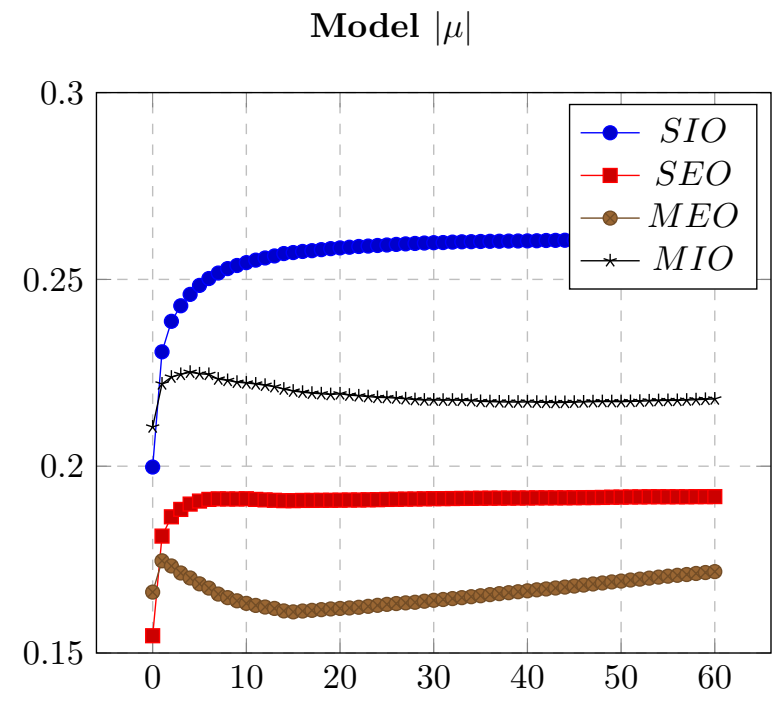

Figure 6: Absolute average of the four models as a function of comparison window.

This indicates the presence of an extreme sale in the time-series.

Investigation into the time series reveals two extreme sales (Teenage Mutant Ninja Turtles \#1 1st edition): a 9.4 sold for $\$ 41$ and a 9.0 sold for $\$ 34.99$. Another sale of a 9.0 three weeks earlier later went for $\$ 4,500$. Examination of the original data source reveals the two sales were in fact for later editions, not first editions. Removing these sales eliminates the jump in variance at 20 days.

This process was used in cleaning the data prior to fitting the models. This case was presented to illustrate how extreme sales may be detected in the time-series. Further work may consider this process as means to detect incorrect sales in the time-series and manually verify values prior to inclusion.

\subsubsection{Selection of Comparison Window}

A 14-day comparison window is selected. Figure 4.3.1 shows the variance of each model as the comparison window varies from 0 to 60 . 


\section{Multiobjectvie Optimization Plot}

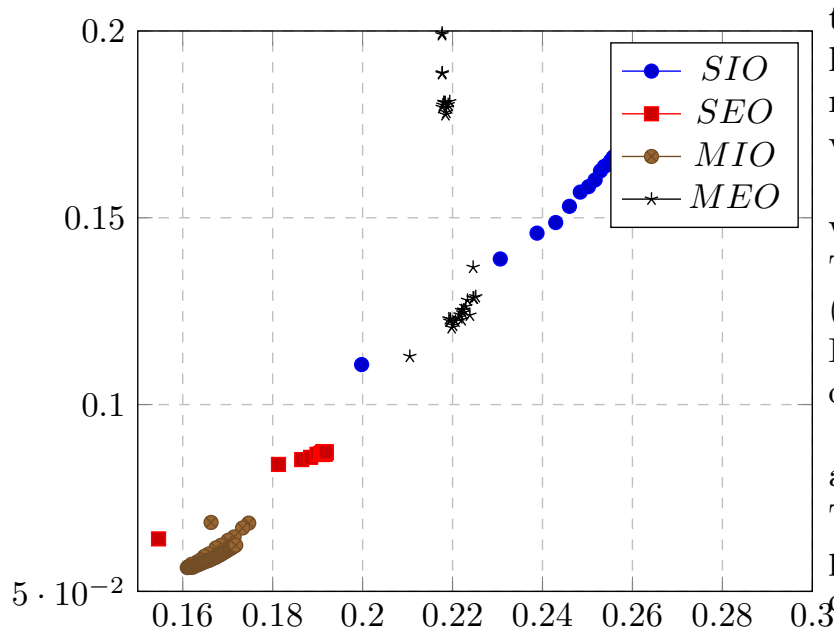

variance of the model $\left(\sigma^{2}=0.084\right)$ and the average of the absolute relative value $(|\mu|=0.20)$. Table 4.3.3 lists several values of the accuracy of the individual model (35\% of predictions are within $10 \%$ of the true value).

In the second case we examine all historical points within the 14-day window and average the results. The variance $\left(\sigma^{2}=0.057\right)$ and absolute relative value $(|\mu|=0.16)$ are improved over the individual model. In this case, $43 \%$ of model predictions are within $10 \%$ of the true value.

Figure 8 show Pearson plots for the distributions arising from the distributions in the average model. The model measures a distribution for every grade pair, and the graph shows these cover a wide range of Pearson families.

\begin{tabular}{|c|c|c|}
\hline \% Relative & Individual & Average \\
\hline 0.01 & 0.045 & 0.050 \\
0.05 & 0.187 & 0.232 \\
0.10 & 0.355 & 0.433 \\
0.15 & 0.500 & 0.600 \\
0.20 & 0.619 & 0.719 \\
0.25 & 0.714 & 0.806 \\
\hline
\end{tabular}

\subsubsection{Analysis of Parameter Choice}

The models are generally insensitive to the choice of parameters in the data selection.

Figure 9 shows a scatter plot comparing two different MIO models. The first model is based on data with a z-score window of 3.00 and uses a 14-day comparison window. The second model has a z-score window of 10.00 and uses a 60-day comparison window.

The relative difference between the models averages $0.24 \%$ across 325 distributions. The correlation coefficient is 0.99 with covariance 5.81 .

Varying the z-score window and comparison window do not strongly affect the MIO model. Insensitivity of the parameters in the data selection process allows these models a wide-range of applicability.

As the comparison window becomes large, the models begin to absorb historical price drift, but up to 60 days the model results are within $1 \%$.

As the z-score window becomes large, outlier sales are included which may bias the model results, but 
Pearson Plot for Distributions for $\mathrm{Z}$-Score $=\mathbf{3 . 0 0}$

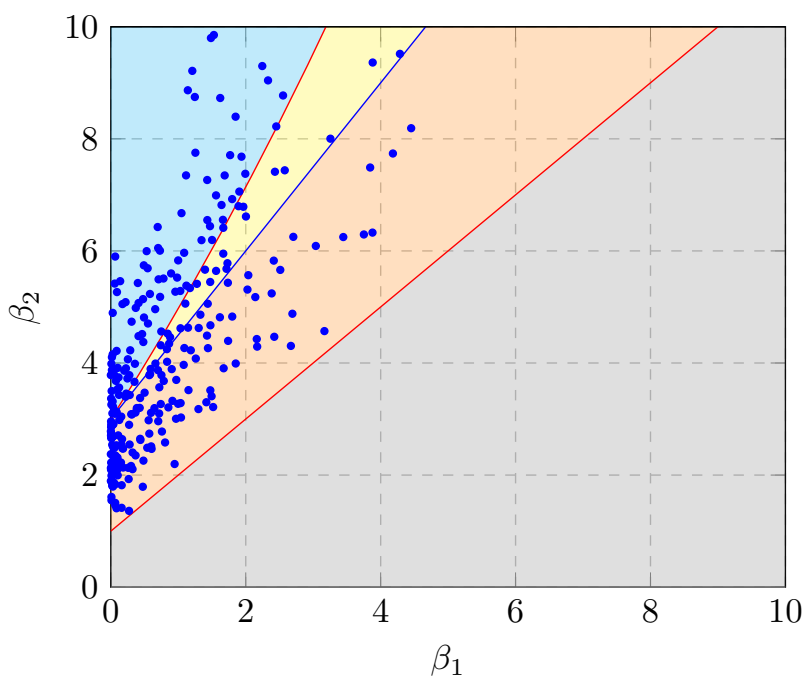

Figure 8: Pearson plot for sales ratios windowed to $\mathrm{z}$-score $=3.00$.

even with a z-score window of 10.00 the effect on the model is minimal.

\subsubsection{Geometric Model}

A Geometric Model uses geometric progression to model the relative values between grades based on the MEO dataset.

Figure 10 plots the relative values between successive grades. From grade 9.2 through 5.0 the relative value remains roughly constant. Lower grades show more variance, but these points are based on only a few data points (4.5-11, 4.0-1, 3.5-2, 2.0-1).

From 9.2 through 5.0 moving up one grade increases the price by and average of $16 \%$. The geometric mean is 0.85 between grades and a comparison between the MEO model (3.00-14) is provided in Table 2.

Figure 11 illustrates one of the difficulties with modeling from the time-series in that the relative values between grades does not always decrease. The values are not constrained together and fit to their corresponding distributions.
MIO Models 3.00 (14) and 10.00 (60)

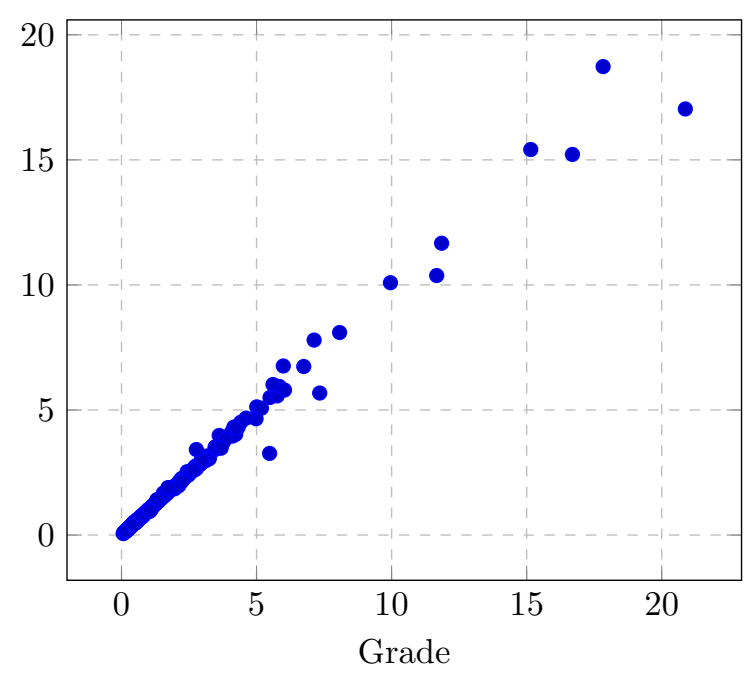

Figure 9: Scatter plot of the model parameters for MIO model 3.00 with a 14-day window and MIO model 10.00 with a 60 -day window.

When comparing grades 9.8 and 2.0, there is only a single sale in the time-series supporting the average value of 0.042 . This value is higher than the relative value between 9.8 and 3.5 (0.033) which means that a 2.0 is prices higher than a 3.5 .

This is not likely true, but the limited data of sales in a 14-day window between a 9.8 and a 2.0 (1), and between a 9.8 and 3.5 (2) provide measurements that are inconsistent. Larger samples are likely to see these values converge to where the 2.0 is prices less than the 3.5 , but the instant time-series does not reflect this.

The Appendix contains a full geometric model based on the MEO(3.00-14) time-series. The model uses averages for grades 1.8 and 1.0 as these are very low frequency and otherwise have unrealistic values.

\subsection{Time-Independent Models}

Time-Independent Models are fit subject to the constraint in 3. The constrained fit is accomplished by first determining the unconstrained model parameters, then determining the best fit subject to the con- 


\section{MEO and Geometric Models}

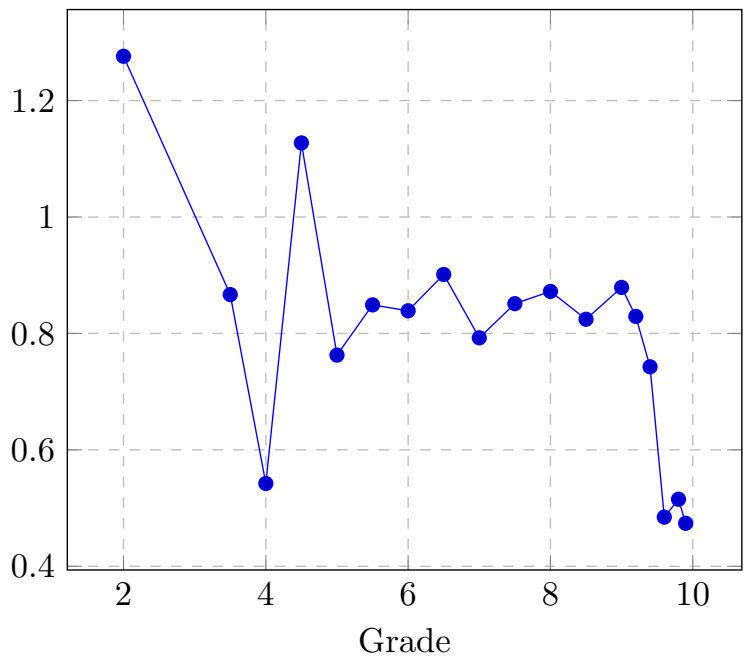

Figure 10: Comparison of MEO model and Geometric model for grades 2.0 through 9.2.

\section{Geometric Model vs. Consistency Model}

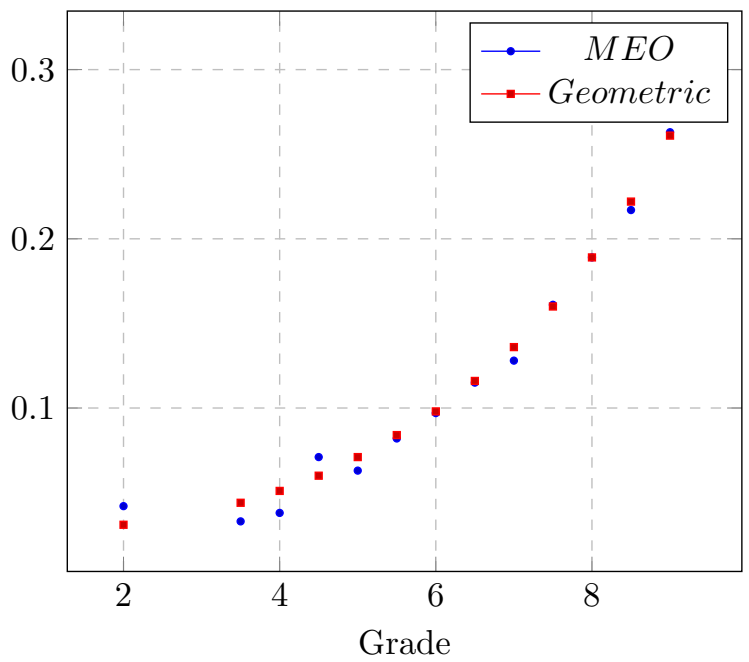

Figure 11: Geometric Model vs. Consistency Model for grades 9.2 and below.

\begin{tabular}{|c|c|c|}
\hline Grade & MEO & Geometric \\
\hline 9.2 & 0.300 & 0.307 \\
\hline 9.0 & 0.263 & 0.261 \\
\hline 8.5 & 0.217 & 0.222 \\
\hline 8.0 & 0.189 & 0.189 \\
\hline 7.5 & 0.161 & 0.160 \\
\hline 7.0 & 0.128 & 0.136 \\
\hline 6.5 & 0.115 & 0.116 \\
\hline 6.0 & 0.097 & 0.098 \\
\hline 5.5 & 0.082 & 0.084 \\
\hline 5.0 & 0.063 & 0.071 \\
\hline 4.5 & 0.071 & 0.060 \\
\hline 4.0 & 0.038 & 0.051 \\
\hline 3.5 & 0.033 & 0.044 \\
\hline 2.0 & 0.042 & 0.031 \\
\hline
\end{tabular}

Table 2: Comparison of Consistency and Geometric Models.

straints.

The time-independent models are not as robust as their time-dependent counterparts. This is in-part because the time-independent models have less independent parameters ( 25 vs. 600 ), but it is also likely these models fail to capture the time-dependent nature of the analysis. Fundamentally the models are intended to predict a future value from a past value, and this inherently incorporates a time-dependence that time-independent models are unable to capture.

From a semantic perspective the fact that timeindependent models are outperformed by timedependent models when applied to time series data seems self-evident. However, it is informative to discuss why a time-depenent model is better suited, and a concrete example may better illustrate the subtle difference.

Suppose we have a past sale in a grade of 9.6, and we wish to predict the fair-market value of a 9.8 . The model factor we employ is $\mathcal{M}(9.8,9.6)$ where we would arrive at the predicted value by multiplying the historical 9.6 sale price by the model factor.

Now suppose we wanted to do the reverse and predict the present fair-market value of a 9.6 based on a recent sale of a 9.8. In this case we would divide the historical 9.8 sale by the model factor $\mathcal{M}(9.6,9.8)$. 
For time-independent models these factors are the same (one multiplies, one divided). With concrete values, suppose the 9.6 previously sold for 100andwepredictthe9.8as200. A time-independent model means that if we recently had a sale of a 9.8 for 200, thenthecurrentpriceofa9.6is 100 . The factor here is 2 (we multiply the 9.6 price by 2 , or divide the 9.8 price by 2 ).

The symmetry displayed here is what is meant by a time-independent model. It doesn't matter which sale comes first, the prices are paired together with the same factor, and we can move between them by multiplying or dividing by this factor.

Time-dependent models are not constrained in this way. In this case we may have a 9.6 sell for 100 andpredicta9.8at200 (factor 2), but a previous sale of a 9.8 at 200 maypredicta9.6valueof 90 (factor $2.2)$.

This is the time-dependence of the model. Prices between grade pairs are not constrained to a single factor, instead each grade pair is decoupled and allowed to vary independently.

The origin of this asymmetry is the time-sequence of the pairs and originates from the influence of one sale on subsequent sales. A previous sale in one grade may increase or decrease the fair-market value in another grade. The time-dependent models are able to capture and quantify this relationship.

\subsubsection{Invalidation of the Time Independent Model}

The constraint 3 provides a means to validate (or invalidate) the time-independent multiplicative model. Independent measurements of the model at various grades may be checked against the constraint, and if the model is valid, the constraints should be satisfied.

The SEO model is used to analyze the validity of the time-independent models because each sales contributes to no more than one model distribution, thus the model parameters are independent of each other.

Figure 12 shows $\mathcal{M}\left(G_{1}, G_{2}\right)$ vs. $\mathcal{M}_{-1}\left(G_{2}, G_{1}\right)$. The average relative difference is $7 \%$ while $\mathcal{M}\left(G_{1}, G_{2}\right)>\mathcal{M}_{-1}\left(G_{2}, G_{1}\right)$ in $75 \%$ of cases. The time-independent constraint is rejected with $P<$ .000001 .

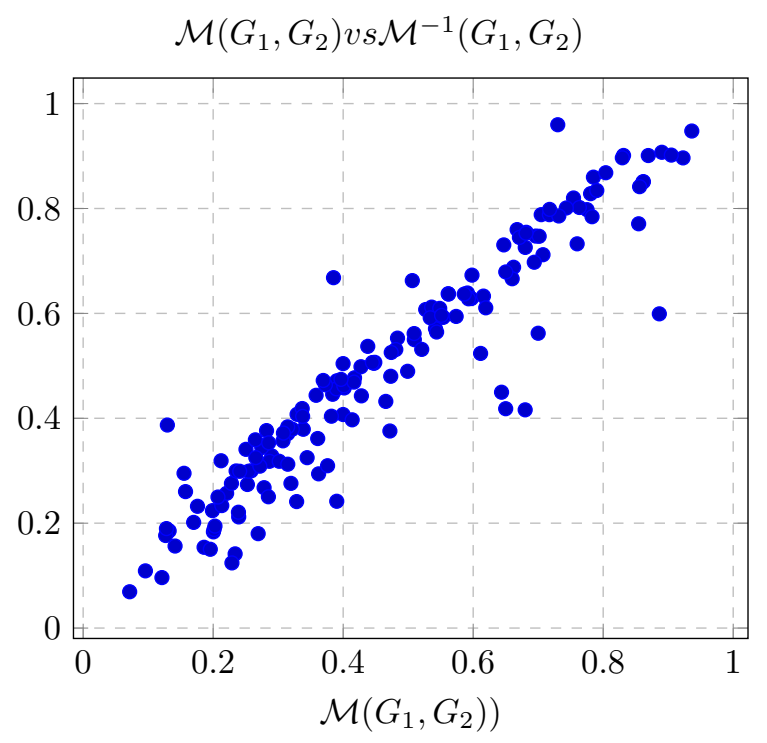

Figure 12: Scatter plot of model parameters for grade pairs.

\subsubsection{Consistency Model}

The Consistency Model forces the constraint from 3 using a Maximum Likelihood method.

Although the constraint is rejected from the previous section, an example of a Consistency Model is provided to illustrate the process of fitting the model with the constraint.

Let $D \in \mathbb{M}$ be a distribution from the unconstrained and let $\mu_{D}$ and $\sigma_{D}$ be the mean and variance of $D$. Let $\mathfrak{V}_{D}$ be the corresponding product of variables corresponding to $D$ in terms of the independent parameters. The likelihood for the Consistency Model is proportional to:

$$
\mathcal{L} \propto \prod_{D \in \mathbb{M}} \exp \left(\frac{1}{2}\left(\frac{\mathfrak{V}_{D}-\mu_{D}}{\sigma_{D}}\right)^{2}\right)
$$

The log-likelihood is easier for computing purposes:

$$
\ln \mathcal{L}=\text { const. }+\sum_{D \in \mathbb{M}} \exp \left(\frac{1}{2}\left(\frac{\mathfrak{V}_{D}-\mu_{D}}{\sigma_{D}}\right)^{2}\right)
$$

The Consistency Model may be computed by maximizing 8. An example spanning tree and Consistency Model are provided in the Appendix. 


\section{$5 \quad$ Predictive Results}

Predictions from MEO (3.00-14) dataset are evaluated, and the individual price predictions average $<0.01 \%\left(\sigma^{2}=0.084\right)$ while the averaged price predictions average $1.1 \%\left(\sigma^{2}=0.057\right)$. The MEO $(3.00-14)$ time-series contains $3,700,902$ individual sales pairs over 251,556 unique sales records.

Predictions are computed using cross-validation. For every sales pair in the MEO dataset, all other pairs containing the sales in the pair are removed. A cross-validation model is computed based on the remaining data, and individual and averaged predictions are computed from the cross-validation model. This process is repeated for every sales pair in the original time-series.

Individual predictions are computed by identifying every prior sale in the 14-day window and computing the relative error in the predicted price. The average relative error is $0.00007\left(\sigma^{2}=0.084\right)$.

Averaged predictions are computed by averaging all individual predictions corresponding to a given sale (from prior sales within the comparison window). The average relative error in this case is 0.011 $\left(\sigma^{2}=0.057\right)$.

The averaged predictions outperformed the individual predictions as seen in Figure 16. The averaged prices see $43.3 \%$ of predictions within $10 \%$ of the true value and $80.6 \%$ of predictions are within $25 \%$.

Figures 13 and 14 provide Pearson plots for distributions of relative and average predictions. For a given predicted grade, a distribution of relative predictions is computed and provided as a single plot in the figure. In both cases the distributions generally follow the Pearson III family with a clustering near Normal.

\section{Observations}

\subsection{Increasing Value of Short-Term Sales}

When two books are sold on the same day at the same grade, the later book has a $5-10 \%$ higher price. This trend reduces to $2-5 \%$ for books sold 1-2 days

\section{Pearson Plot Relative Predictions MEO} (3.00-14)

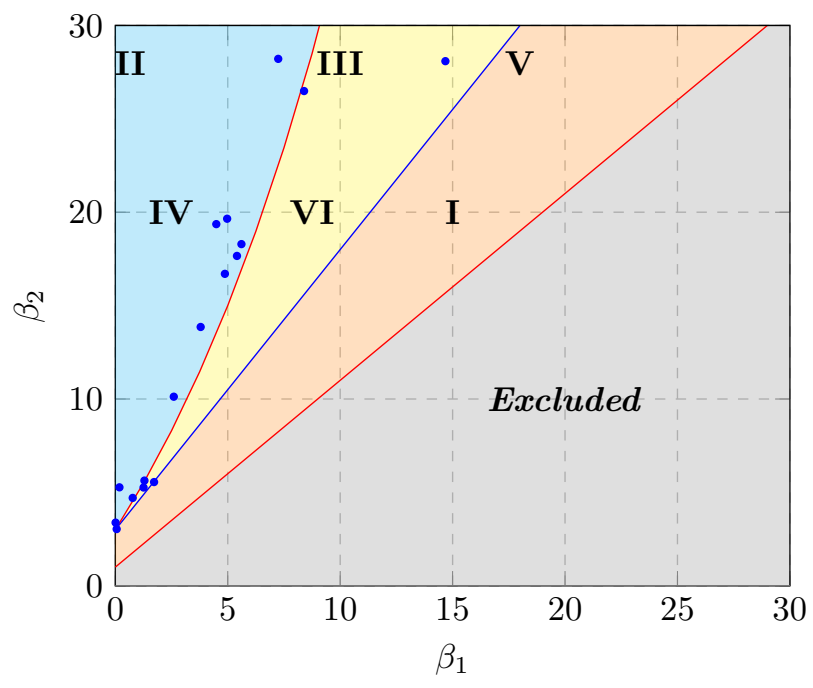

Figure 13: Pearson plot for the distributions of relative predicted prices based on the $\mathrm{MEO}(3.00-14)$ time-series.

apart and vanishes after 3 days.

Figure 17 shows the increased value over each grade by day. For books sold on the same day, there is an increased price for the later book across every grade. The average across all grades is $8 \%$ higher for the later book.

At one day apart, the later book sees an average $3.3 \%$ higher price while after two days we see an average $2.1 \%$ higher price. At three days apart the later book holds a $1.4 \%$ advantage while the trend decreases to below $1 \%$ after three days.

\subsubsection{Sawtooth Pattern Over Grades}

There is a distinct pattern between the relative prices of books between consecutive grades. Figure 18 illustrates one example of the saw-tooth pattern appearing in the relative prices between grades.

When the primary grade increases (i.e. 7.5 to 8.0 ) there is on average a $20 \%$ increase in price. However, when the primary grade remains the same (i.e. 8.0 to 8.5 ) the is on average a $7.5 \%$ increase in price. 
Pearson Plot Average Predictions MEO (3.00-14)

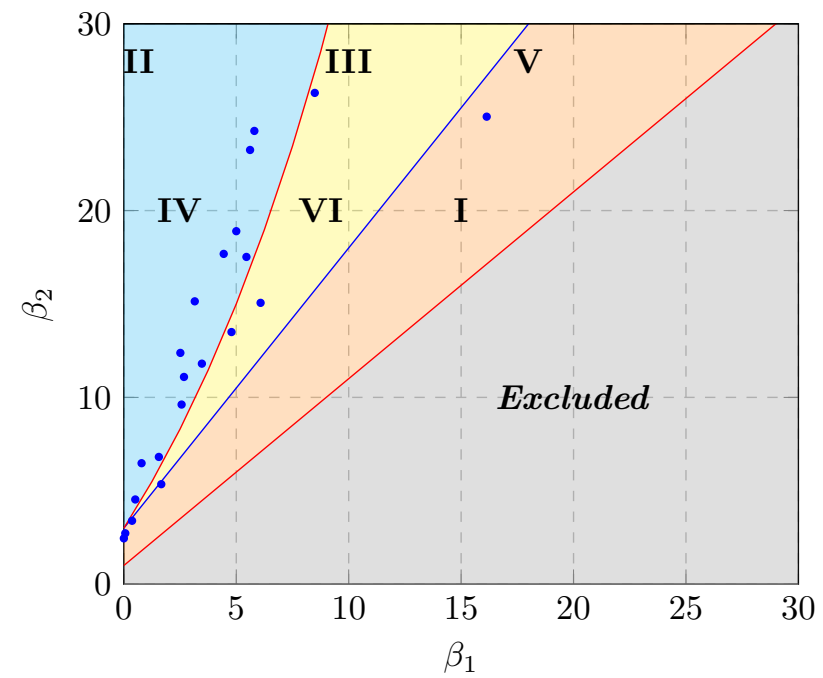

Figure 14: Pearson plot for the distributions of averaged predicted prices based on the MEO(3.00-14) time-series.

\section{Predicted Prices MEO (3.00-14)}

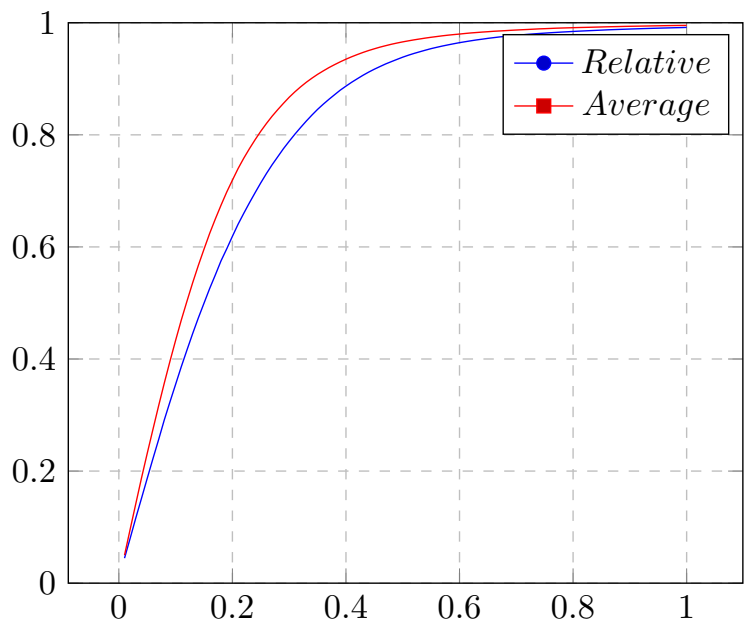

Figure 15: Percent of predicted sale prices within relative price.

\section{Cumulative Distribution of Predicted Prices MEO (3.00-14)}

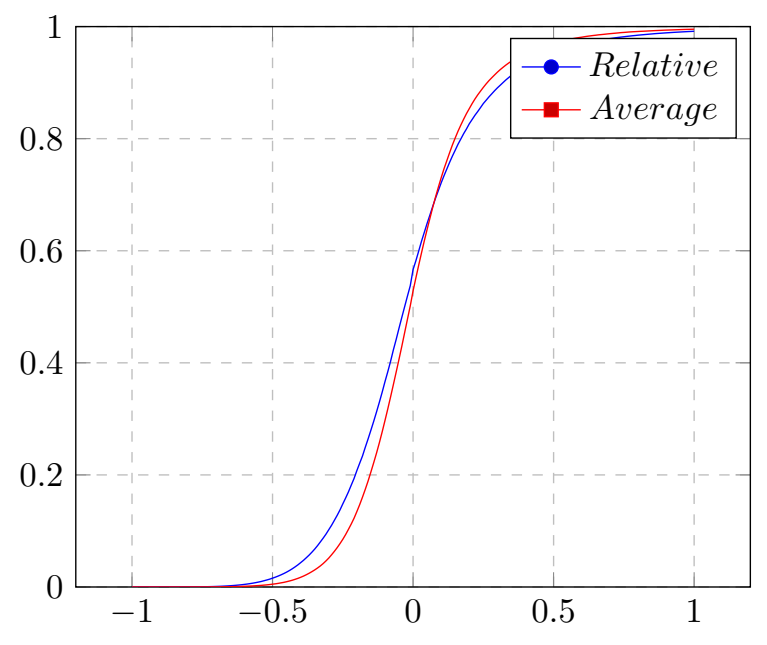

Figure 16: Cumulative distribution of predicted sale prices for MEO (3.00-14).

\section{Books Sold at Same Grade}

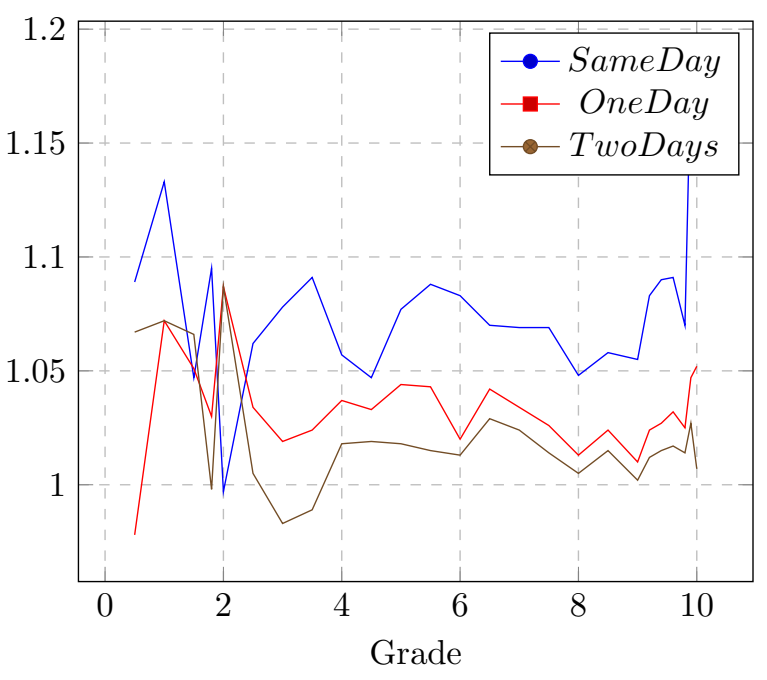

Figure 17: Books sold later show an increased price with the trend diminishing over time. 


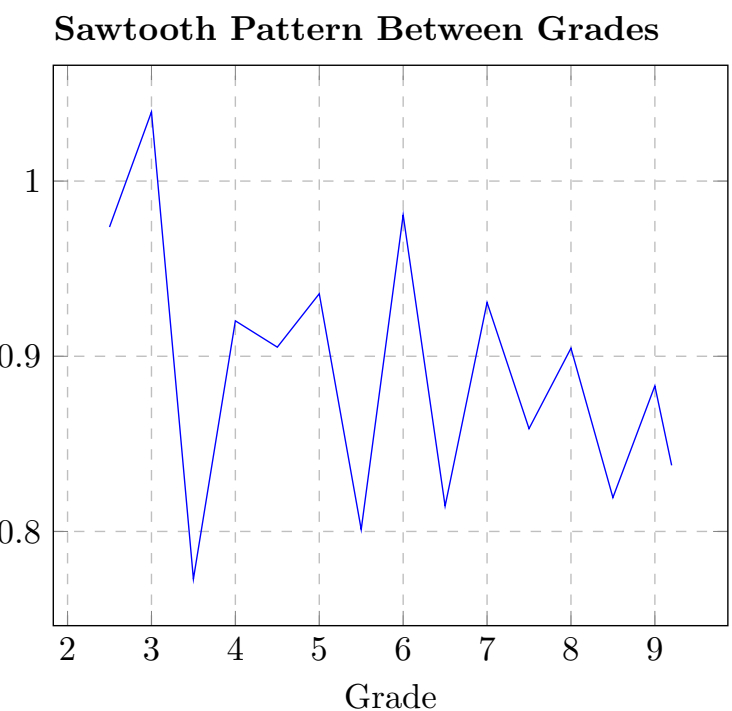

Figure 18: Books sold later show an increased price with the trend diminishing over time.

The larger price increase moving up a primary grade may originate in the desirability of higher leading numbers. Moving from 7.5 to 8.0 has a larger appear than moving from a 7.0 to 7.5 .

\section{Conclusion}

We analyzed several time-series for comic sales and constructed many models to predict future sales based on past sales. The Multi-Paired Exclude Orphan model with a 3.00 z-score window for data cleansing and a 14-day comparison window for grouping sales into pairs.

The individual sales accurately predicted the future price to within $10 \%$ on $35 \%$ of attempts while the average method accomplished $43 \%$ within $10 \%$ of true value. These numbers rise quickly with $62 \%$ of individual predictions within $20 \%$ of true value, and $72 \%$ of averaged predictions with $20 \%$ of true value.

The analysis produced 1,220 different models by examining the SIO, SEO, MIO, and MEO datasets at five different z-score windows and 61 different comparison windows. Predictions from each of these models were evaluated by cross-validation which requires producing a model to test each sales pair producing 1,102,575,644 cross-validation models.

Models are generally insensitive to the selection of z-score and comparison windows. MEO models are the best predictors, followed by SEO models, then MIO models, and finally SIO models.

Topics for future work include

Platform Sensitivity Different sales platforms charge different fees. How much does this affect the sale price?

Compare Grading Companies There is a belief in the market that CGC books are priced higher at the same grade. It would be beneficial to quantify this.

CGC Census Data Can the CGC Census data help refine the prices?

\section{Identify Incorrect/Fraudulent Transactions} During this research we identified many incorrect transactions based on the effect they had on the variance of our time-series.

\section{References}

[1] All-time record comic book sales, 2021.

[2] Argosy price guide 1, 2021.

[3] Comic book market 2021: Industry analysis, size, share, revenue, prominent players, developing technologies, tendencies and forecasts to 2027 with dominant sectors and countries data, 2021.

[4] Comic book price guide, 2021.

[5] Comics buyer's guide, 2021.

[6] Standard catalog of comic books, 2021.

[7] Wizard: The guide to comics, 2021.

[8] F. Anowar and S. Sadaoui. Detection of auction fraud in commercial sites. Journal of theoretical and applied electronic commerce research, 2020. 
[9] Arthur Cayley. A theorem on trees. Quart. J. Pure Appl. Math, 23, 1899.

[10] S. M. Shats F. Dong and H. Xu. Reasoning under uncertainty for shill detection in online auctions using dempster-shafer theory. International Journal of Software Engineering and Knowledge Engineering, 2010.

[11] J. B. Kruskal. On the shortest spanning subtree of a graph and the traveling salesman problem. Proceedings of the American Mathematical Society, 7, 1956.

[12] J. Trevathan N. Majadi and H. Gray. A runtime algorithm for detecting shill bidding in online auctions. Journal of theoretical and applied electronic commerce research, 2018.

[13] Karl Pearson. Contributions to the mathematical theory of evolution. Proceedings of the Royal Society, 54, 1893.

[14] Hassler Whitney. On the abstract properties of linear dependence. American Journal of Mathematics, 57, 1935.

[15] T. Yoshida and H. Ohwada. Shill bidder detection for online auctions. Proceedings of the 11th Pacific Rim International Conference on Artificial Intelligence, 2010.

\section{Appendix A}

This Appendix provided several model tables for ease of reference. Historical grades are read down the rows while predicted grades are read across. Cell values are a multiplier against the historical grade to compute the predicted future grade. 

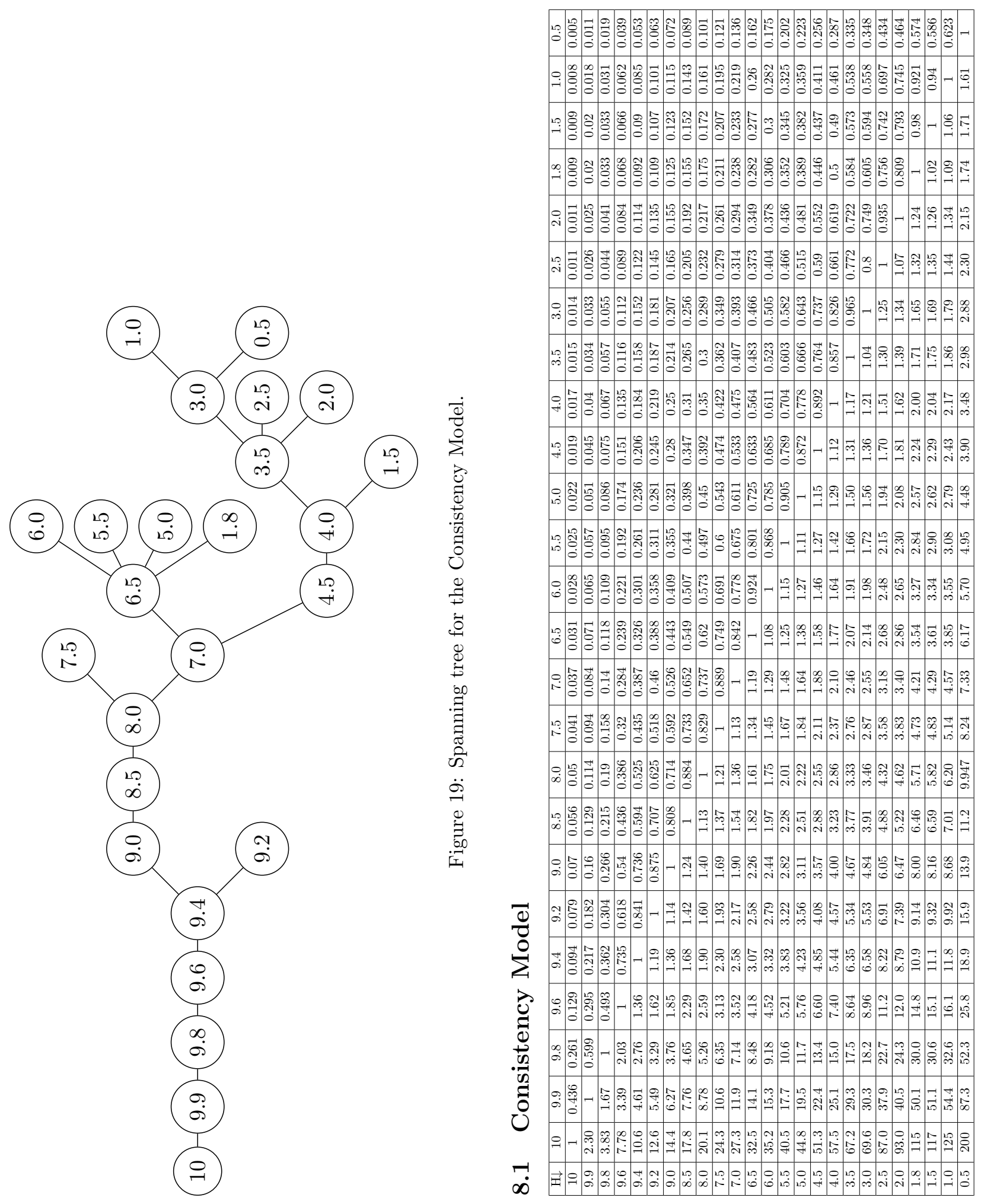


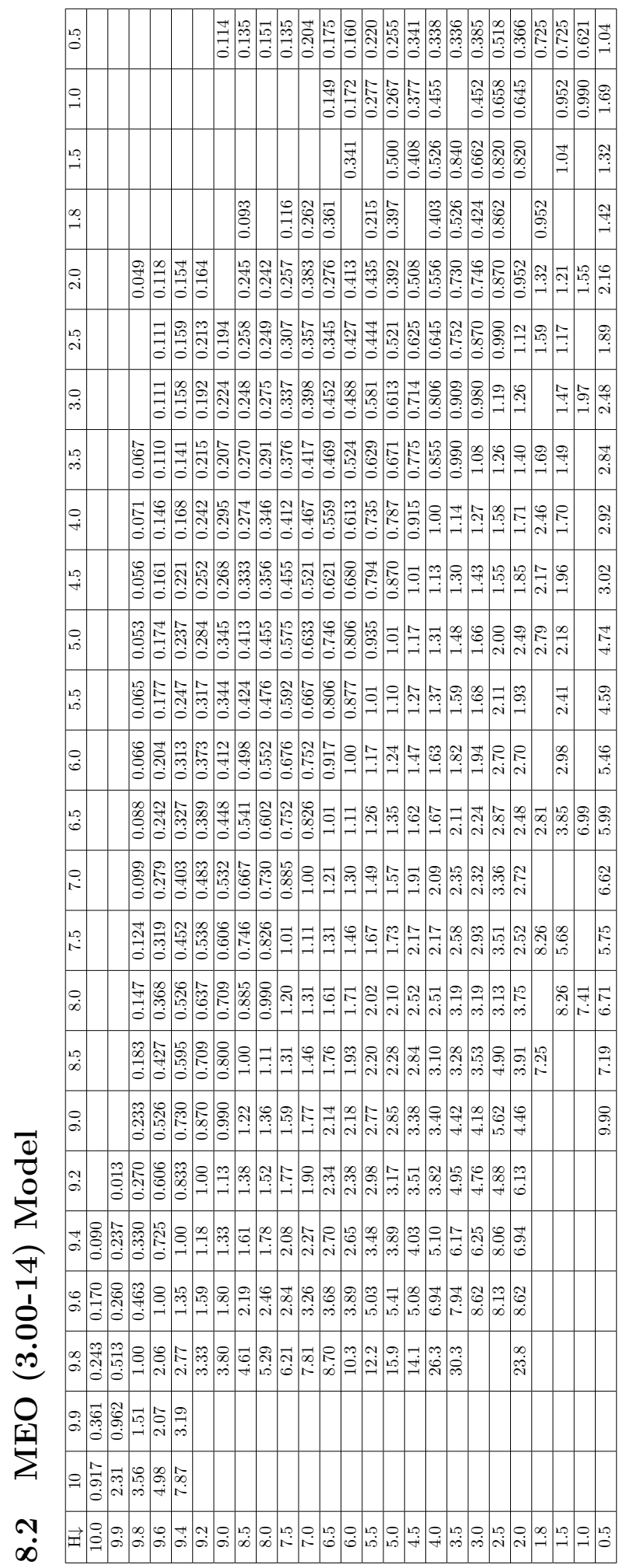

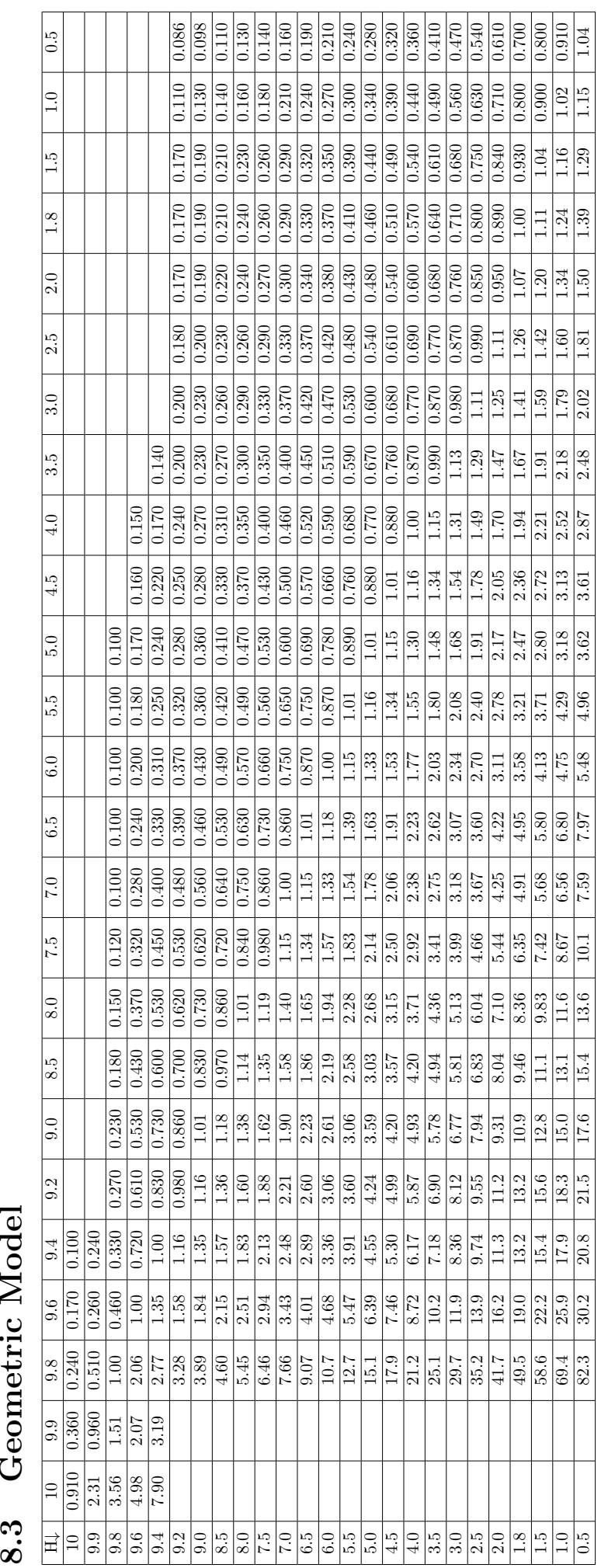

